\title{
Borel Directions and Uniqueness of Meromorphic Functions
}

\author{
Keyu Zhang, ${ }^{1,2}$ HongYan $\mathrm{Xu}^{3}$ and Hongxun $\mathrm{Yi}^{1}$ \\ ${ }^{1}$ School of Mathematics, Shandong University, Jinan, Shandong 250100, China \\ ${ }^{2}$ Department of Mathematics, Qilu Normal University, Jinan, Shandong 250013, China \\ ${ }^{3}$ Department of Informatics and Engineering, Jingdezhen Ceramic Institute, Jingdezhen, Jiangxi 333403, China
}

Correspondence should be addressed to Keyu Zhang; sduzky@163.com

Received 15 April 2013; Accepted 23 July 2013

Academic Editor: Paul Eloe

Copyright (C) 2013 Keyu Zhang et al. This is an open access article distributed under the Creative Commons Attribution License, which permits unrestricted use, distribution, and reproduction in any medium, provided the original work is properly cited.

We investigate the relationship between Borel directions and uniqueness of meromorphic functions and obtain some results of meromorphic functions sharing four distinct values IM and one set in an angular domain containing a Borel line. Our result is an improvement of a recent theorem given by Long and $\mathrm{Wu}$ (2012).

\section{Introduction and Main Results}

We use $\mathbb{C}$ to denote the open complex plane, $\widehat{\mathbb{C}}(=\mathbb{C} \bigcup\{\infty\})$ to denote the extended complex plane, and $\Omega$ (C $\mathbb{C}$ ) to denote an angular domain. The fundamental results and the standard notations of the Nevanlinna value distribution theory of meromorphic functions will be used in $[1,2]$. In addition, the order of meromorphic function $f$ is defined by

$$
\rho=\rho(f)=\limsup _{r \rightarrow \infty} \frac{\log T(r, f)}{\log r} .
$$

Let $S$ be a set of distinct elements in $\widehat{\mathbb{C}}$ and $\Omega:=\{z: \alpha \leq$ $\arg z \leq \beta\} \subseteq \mathbb{C}$. Define

$$
\begin{aligned}
E & (S, \Omega, f) \\
& =\bigcup_{a \in S}\left\{z \in \Omega \mid f_{a}(z)=0, \text { counting multiplicities }\right\}, \\
\bar{E} & (S, \Omega, f) \\
& =\bigcup_{a \in S}\left\{z \in \Omega \mid f_{a}(z)=0, \text { ignoring multiplicities }\right\},
\end{aligned}
$$

where $f_{a}(z)=f(z)-a$ if $a \in \mathbb{C}$ and $f_{\infty}(z)=1 / f(z)$.

Let $f$ and $g$ be two nonconstant meromorphic functions in $\mathbb{C}$. If $E(S, \Omega, f)=E(S, \Omega, g)$, then we say $f$ and $g$ share the set $S$ CM (counting multiplicities) in $\Omega$. If $\bar{E}(S, \Omega, f)=$ $\bar{E}(S, \Omega, g)$, then we say $f$ and $g$ share the set $S$ IM (ignoring multiplicities) in $\Omega$. In particular, when $S=\{a\}, a \in \widehat{\mathbb{C}}$, we say $f$ and $g$ share the value $a$ CM in $\Omega$ if $E(S, \Omega, f)=$ $E(S, \Omega, g)$, and we say $f$ and $g$ share the value $a$ IM in $\Omega$ if $\bar{E}(S, \Omega, f)=\bar{E}(S, \Omega, g)$. When $\Omega=\mathbb{C}$, we give the simple notation as before, $E(S, f), \bar{E}(S, f)$, and so on (see [3]).

Nevanlinna (see [4]) proved the following well-known theorems.

Theorem 1 (see [4]). If $f$ and $g$ are two nonconstant meromorphic functions that share five distinct values $a_{1}, a_{2}, a_{3}, a_{4}, a_{5}$ IM in $\mathbb{C}$, then $f(z) \equiv g(z)$.

Theorem 2 (see [4]). If $f$ and $g$ are two distinct nonconstant meromorphic functions that share four distinct values $a_{1}, a_{2}, a_{3}, a_{4} C M$ in $\mathbb{C}$, then $f$ is a Möbius transformation of $g$, two of the shared values, say $a_{1}$ and $a_{2}$, are Picard values, and the cross ratio $\left(a_{1}, a_{2}, a_{3}, a_{4}\right)=-1$.

After their very work, many investigations studied the uniqueness of meromorphic functions with shared values in the whole complex plane (see [5]). Zheng studied the uniqueness problem under the condition that five values and four values are shared in some angular domain in $\mathbb{C}$ around 2003 (see [6, 7]). It is an interesting topic to investigate the uniqueness with shared values in the angular domain; 
see $[3,6-12]$. The basic notations and definitions of meromorphic functions in an angular domain will be introduced as follows (see $[1,6,7]$ ).

Let $f$ be a meromorphic function on the angular domain $\Omega(\alpha, \beta)=\{z: \alpha \leq \arg z \leq \beta\}$ and $0<\beta-\alpha \leq 2 \pi$. Define

$$
\begin{aligned}
& A_{\alpha, \beta}(r, f)=\frac{\omega}{\pi} \int_{1}^{r}\left(\frac{1}{t^{\omega}}-\frac{t^{\omega}}{r^{2 \omega}}\right) \\
& \quad \times\left\{\log ^{+}\left|f\left(t e^{i \alpha}\right)\right|+\log ^{+}\left|f\left(t e^{i \beta}\right)\right|\right\} \frac{d t}{t}, \\
& B_{\alpha, \beta}(r, f)=\frac{2 \omega}{\pi r^{\omega}} \int_{\alpha}^{\beta} \log ^{+}\left|f\left(r e^{i \theta}\right)\right| \sin \omega(\theta-\alpha) d \theta \\
& C_{\alpha, \beta}(r, f)=2 \sum_{1<\left|b_{\mu}\right|<r}\left(\frac{1}{\left|b_{\mu}\right|^{\omega}}-\frac{\left|b_{\mu}\right|^{\omega}}{r^{2 \omega}}\right) \sin \omega\left(\theta_{\mu}-\alpha\right), \\
& S_{\alpha, \beta}(r, f)=A_{\alpha, \beta}(r, f)+B_{\alpha, \beta}(r, f)+C_{\alpha, \beta}(r, f),
\end{aligned}
$$

where $\omega=\pi /(\beta-\alpha)$ and $b_{\mu}=\left|b_{\mu}\right| e^{i \theta_{\mu}}(\mu=1,2, \ldots)$ are the poles of $f$ on $\Omega(\alpha, \beta)$ counted according to their multiplicities. $S_{\alpha, \beta}(r, f)$ is called the Nevanlinna's angular characteristic, and $C_{\alpha, \beta}(r, f)$ is called the angular counting function of the poles of $f$ on $\Omega(\alpha, \beta)$, and $\bar{C}_{\alpha, \beta}(r, f)$ is the reduced function of $C_{\alpha, \beta}(r, f)$. Similarly, when $a \neq \infty$, we will use the notations $A_{\alpha, \beta}(r, 1 /(f-a)), B_{\alpha, \beta}(r, 1 /(f-a))$, $C_{\alpha, \beta}(r, 1 /(f-a)), S_{\alpha, \beta}(r, 1 /(f-a))$, and so on.

It is well known that angular distribution is an interesting topic of value distribution of meromorphic function in complex analysis, and Borel directions played an important role in the topic of angular distribution (see [13-24]). Valiron [16] proved that every meromorphic function of finite order $\rho>0$ has at least one Borel direction of order $\rho$. Chuang [25] investigated the existence of Borel direction of meromorphic function of infinite order. To state the Chuang's results, we will introduce the definition as follows.

Definition 3 (see [25]). Let $f$ be a meromorphic function of infinite order, $\rho(r)$ is a real function satisfying the following conditions:

(i) $\rho(r)$ is continuous, nondecreasing for $r \geq r_{0}$ and $\rho(r) \rightarrow \infty$ as $r \rightarrow \infty$;

(ii)

$$
\lim _{r \rightarrow \infty} \frac{\log U(R)}{\log U(r)}=1, \quad R=r+\frac{r}{\log U(r)},
$$

where $U(r)=r^{\rho(r)}\left(r \geq r_{0}\right)$;

(iii)

$$
\limsup _{r \rightarrow \infty} \frac{\log T(r, f)}{\log U(r)}=1
$$

Then $\rho(r)$ is called infinite order of meromorphic function $f$. This definition is given by Chuang [25].
Let $\rho(r)$ be infinite order of meromorphic function $f$; we will denote by $M(\rho(r))$ the set of meromorphic function $g$ satisfying $\lim \sup _{r \rightarrow \infty}(\log T(r, g) / \rho(r) \log r) \leq 1$; that is,

$$
M(\rho(r)):=\left\{g: \limsup _{r \rightarrow \infty} \frac{\log T(r, g)}{\rho(r) \log r} \leq 1\right\} .
$$

Let $\alpha<\beta, \beta-\alpha<2 \pi, r>0$, and $\Omega(\alpha, \beta, r):=\{z$ : $\alpha \leq \arg z \leq \beta, 0<|z| \leq r\}$. The definition of Borel direction of meromorphic functions $f$ of infinite order $\rho(r)$ is given as follows.

Definition 4 (see [25]). Let $f$ be meromorphic functions of infinite order $\rho(r)$; if for any $\varepsilon(0<\varepsilon<\pi)$, the equality

$$
\limsup _{r \rightarrow \infty} \frac{\log n(\Omega(\theta-\varepsilon, \theta+\varepsilon, r), f=a)}{\rho(r) \log r}=1
$$

holds for any complex number $a \in \widehat{\mathbb{C}}$, at most except two exceptions, where $n(\Omega(\theta-\varepsilon, \theta+\varepsilon, r), f=a)$ is the counting function of zeros of the function $f-a$ in the angular domain $\Omega(\theta-\varepsilon, \theta+\varepsilon)$, counting multiplicities, then the ray $\arg z=$ $\theta$ is called a Borel direction of $\rho(r)$ order of meromorphic function $f$.

Remark 5. Chuang [25] proved that every meromorphic function $f$ of infinite order $\rho(r)$ has at least one Borel direction of infinite order $\rho(r)$.

In 2012, Long and $\mathrm{Wu}$ [26] investigated the problem concerning Borel direction and shared value of meromorphic functions and obtained the following theorems.

Theorem 6 (see [26, Theorem 1.1]). Let $f$ be a meromorphic function of infinite order $\rho(r)$, and let $g \in M(\rho(r))$, $\arg z=$ $\theta(0 \leq \theta<2 \pi)$ be one Borel direction of $\rho(r)$ order of meromorphic function $f$; let $a_{i} \in \widehat{\mathbb{C}}(i=1,2,3,4,5)$ be five distinct complex numbers. If $f$ and $g$ share $a_{i}(i=1,2,3,4,5)$ IM in the angular domain $\Omega(\theta-\varepsilon, \theta+\varepsilon)$ for any $\varepsilon(0<\varepsilon<\pi)$, then $f \equiv g$.

Theorem 7 (see [26, Theorem 1.2]). Let $f$ be a meromorphic function of infinite order $\rho(r)$, and let $g \in M(\rho(r))$, $\arg z=$ $\theta(0 \leq \theta<2 \pi)$ be one Borel direction of $\rho(r)$ order of meromorphic function $f$; let $a_{i} \in \widehat{\mathbb{C}}(i=1,2,3,4)$ be four distinct complex numbers. If $f$ and $g$ share $a_{i}(i=1,2,3,4) C M$ in the angular domain $\Omega(\theta-\varepsilon, \theta+\varepsilon)$ for any $\varepsilon(0<\varepsilon<\pi)$, then $f$ is a Möbius transformation of $g$.

Thus, a question arises naturally: could the nature of sharing the values $\alpha_{i}$ be further relaxed in Theorems 6 and 7?

In this paper, we will deal with the above question and obtain the following result which is an improvement of Theorem 6 .

Theorem 8. Let $f$ be a meromorphic function of infinite order $\rho(r)$, and let $g \in M(\rho(r))$, arg $z=\theta(0 \leq \theta<2 \pi)$ be one Borel direction of $\rho(r)$ order of meromorphic function $f$; we assume that $f$ and $g$ share four distinct values $a_{j}(j=1,2,3,4) I M$ in 
$\Omega(\theta-\varepsilon, \theta+\varepsilon)$, and $\bar{E}(S, \Omega(\theta-\varepsilon, \theta+\varepsilon), f) \subset \bar{E}(S, \Omega(\theta-\varepsilon, \theta+$ $\varepsilon), g)$, for any $\varepsilon(0<\varepsilon<\pi)$, where $S=\left\{b_{1}, \ldots, b_{m}\right\}, m \geq 1$ and $b_{1}, \ldots, b_{m} \in \widehat{\mathbb{C}} \backslash\left\{a_{1}, a_{2}, a_{3}, a_{4}\right\}$. Then $f$ and $g$ share all values CM; thus, it follows that either $f \equiv g$ or $f$ is a Möbius transformation of $g$. Furthermore, if the number of the values in $S$ is odd, then $f \equiv g$.

Remark 9. The special case $m=1$ of this theorem immediately yields Theorem 6 . In fact, when $m=1$, set $S=\left\{a_{5}\right\}$. If $f, g$ share $a_{5}$ IM, which implies $\bar{E}(S, \Omega(\theta-\varepsilon, \theta+\varepsilon), f) \subset$ $\bar{E}(S, \Omega(\theta-\varepsilon, \theta+\varepsilon), g)$, then by Theorem 8 , we can get $f \equiv g$.

\section{Some Lemmas}

To prove our result, we require the following lemmas.

Lemma 10 (see [27]). Let $f$ be a nonconstant meromorphic function on $\Omega(\alpha, \beta)$. Then for arbitrary complex number $a$, we have

$$
S_{\alpha, \beta}\left(r, \frac{1}{f-a}\right)=S_{\alpha, \beta}(r, f)+\varepsilon(r, a),
$$

where $\varepsilon(r, a)=O(1)$ as $r \rightarrow \infty$.

Lemma 11 (see $[7,28]$ ). Suppose that $f$ is a nonconstant meromorphic function in one angular domain $\Omega(\alpha, \beta)$ with $0<$ $\beta-\alpha \leq 2 \pi$; then, for arbitrary $q$ distinct $a_{j} \in \widehat{\mathbb{C}}(1 \leq j \leq q)$, we have

$$
(q-2) S_{\alpha, \beta}(r, f) \leq \sum_{j=1}^{q} \bar{C}_{\alpha, \beta}\left(r, \frac{1}{f-a_{j}}\right)+R_{\alpha, \beta}(r, f),
$$

where the term $\bar{C}_{\alpha, \beta}\left(r, 1 /\left(f-a_{j}\right)\right)$ will be replaced by $\bar{C}_{\alpha, \beta}(r, f)$ when some $a_{j}=\infty$ and

$$
\begin{aligned}
R_{\alpha, \beta}(r, f)= & A_{\alpha, \beta}\left(r, \frac{f^{\prime}}{f}\right)+B_{\alpha, \beta}\left(r, \frac{f^{\prime}}{f}\right) \\
& +\sum_{j=1}^{q}\left\{A_{\alpha, \beta}\left(r, \frac{f^{\prime}}{f-a_{j}}\right)+B_{\alpha, \beta}\left(r, \frac{f^{\prime}}{f-a_{j}}\right)\right\} \\
& +O(1) .
\end{aligned}
$$

Lemma 12 (see [27, Page 138]). Let $f$ be a nonconstant meromorphic function in the whole complex plane $\mathbb{C}$. Given one angular domain on $\Omega(\alpha, \beta)$. Then, for any $1 \leq r<R$, we have

$$
\begin{gathered}
A_{\alpha, \beta}\left(r, \frac{f^{\prime}}{f}\right) \leq K\left\{\left(\frac{R}{r}\right)^{\omega} \int_{1}^{R} \frac{\log ^{+} T(r, f)}{t^{1+\omega}} d t\right. \\
\left.+\log ^{+} \frac{r}{R-r}+\log \frac{R}{r}+1\right\}, \\
B_{\alpha, \beta}\left(r, \frac{f^{\prime}}{f}\right) \leq \frac{4 \omega}{r^{\omega}} m\left(r, \frac{f^{\prime}}{f}\right),
\end{gathered}
$$

where $\omega=\pi /(\beta-\alpha)$ and $K$ is a positive constant not depending on $r$ and $R$.
Remark 13. Nevanlinna conjectured that

$$
A_{\alpha, \beta}\left(r, \frac{f^{\prime}}{f}\right)+B_{\alpha, \beta}\left(r, \frac{f^{\prime}}{f}\right)=o\left(S_{\alpha, \beta}(r, f)\right)
$$

when $r$ tends to $+\infty$ outside an exceptional set of finite linear measure, and he proved that $A_{\alpha, \beta}\left(r, f^{\prime} / f\right)+B_{\alpha, \beta}\left(r, f^{\prime} / f\right)=$ $O(1)$ when the function $f$ is meromorphic in $\mathbb{C}$ and has finite order. In 1975, Goldberg [28] constructed a counter example to show that (13) is not valid.

Remark 14. From Lemmas 11 and 12, we can get the following conclusion:

$$
R_{\alpha, \beta}(r, f)= \begin{cases}O(1), & f \text { is of finite order, } \\ O(\log U(r)), r \notin E, & f \text { is of infinite order, }\end{cases}
$$

where $R_{\alpha, \beta}(r, f)$ is stated as in (10), $U(r)=r^{\rho(r)}, \rho(r)$ is infinite order of meromorphic function $f$, and $E$ is a set of finite linear measure.

Remark 15. From the definition of $A_{\alpha, \beta}(r, f), B_{\alpha, \beta}(r, f)$, $C_{\alpha, \beta}(r, f), S_{\alpha, \beta}(r, f)$, and Lemmas 10-12, we can see that the properties of $C_{\alpha, \beta}(r, f),(A+B)_{\alpha, \beta}(r, f)$, and $S_{\alpha, \beta}(r, f)$ are the same as for the more familiar quantities $N(r, f), m(r, f)$, and $T(r, f)$, respectively.

Lemma 16 (see [29]). Let $f$ be meromorphic function of infinite order $\rho(r)$. Then the ray $\arg z=\theta$ is one Borel direction of $\rho(r)$ order of meromorphic function $f$ if and only if $f$ satisfies the equality

$$
\limsup _{r \rightarrow \infty} \frac{\log S_{\theta-\epsilon, \theta+\epsilon}(r, f)}{\rho(r) \log r}=1
$$

for any $\epsilon(0<\epsilon<\pi / 2)$.

By using the same argument as in [8, Lemma 1] and [5], we can get the lemma below easily.

Lemma 17. Suppose that $f$ is a nonconstant meromorphic function with infinite order $\rho(r)$, and the ray $\arg z=\theta$ is one Borel direction of $\rho(r)$ order of meromorphic function $f$. Let $P(f)=a_{0} f^{p}+a_{1} f^{p-1}+\cdots+a_{p}\left(a_{0} \neq 0\right)$ be a polynomial of $f$ with degree $p$, where the coefficients $a_{j}(j=0,1, \ldots, p)$ are constants, and let $b_{j}(j=1,2, \ldots, q)$ be $q(q \geq p+1)$ distinct finite complex numbers. Then for any $\varepsilon(0<\varepsilon<\pi / 2)$,

$$
\begin{aligned}
(A+B)_{\theta-\varepsilon, \theta+\varepsilon} & \left(r, \frac{P(f) \cdot f^{\prime}}{\left(f-b_{1}\right)\left(f-b_{2}\right) \cdots\left(f-b_{q}\right)}\right) \\
& =R_{\theta-\varepsilon, \theta+\varepsilon}(r, f) .
\end{aligned}
$$

Lemma 18. Suppose that $f$ is a nonconstant meromorphic function with infinite order $\rho(r)$, and the ray $\arg z=\theta$ is one Borel direction of $\rho(r)$ order of meromorphic function $f$. We assume that for any for any $\varepsilon(0<\varepsilon<\pi / 2), f$ and $g$ share 
four distinct values $a_{j}(j=1,2,3,4) I M$ in angular domain $\Omega(\theta-\varepsilon, \theta+\varepsilon)$ and $f \neq \equiv$ g. Let

$$
\begin{aligned}
\Psi=f^{\prime} g^{\prime}(f-g)^{2} \times( & \left(f-a_{1}\right)\left(f-a_{2}\right)\left(f-a_{3}\right)\left(f-a_{4}\right) \\
& \times\left(g-a_{1}\right)\left(g-a_{2}\right) \\
& \left.\times\left(g-a_{3}\right)\left(g-a_{4}\right)\right)^{-1} .
\end{aligned}
$$

Then, $S_{\theta-\varepsilon, \theta+\varepsilon}(r, \Psi)=R_{\theta-\varepsilon, \theta+\varepsilon}(r, f)+R_{\theta-\varepsilon, \theta+\varepsilon}(r, g)$.

Proof. Since the ray $\arg z=\theta$ is one Borel direction of meromorphic function $f$ of $\rho(r)$ order, thus, for any $\varepsilon(0<$ $\varepsilon<\pi / 2)$, we can assume that $z_{0} \in \Omega(\theta-\varepsilon, \theta+\varepsilon)$ and $f\left(z_{0}\right)=a_{1}$ (or $a_{2}, a_{3}, a_{4}$ ) with multiplicity $p$ and $g\left(z_{0}\right)=a_{1}$ (or $a_{2}, a_{3}, a_{4}$ ) with multiplicity $q$. From (17), we can get

$$
\Psi(z)=O\left(\left(z-z_{0}\right)^{2 \min (p, q)-2}\right) .
$$

Hence, $\Psi$ is analytic in $\Omega(\theta-\varepsilon, \theta+\varepsilon)$. By Lemmas 12 and 17 , we have

$$
\begin{aligned}
& S_{\theta-\varepsilon, \theta+\varepsilon}(r, \Psi)=(A+B)_{\theta-\varepsilon, \theta+\varepsilon}(r, \Psi) \\
& \leq(A+B)_{\theta-\varepsilon, \theta+\varepsilon} \\
& \times\left(r, \frac{f^{\prime}}{\left(f-a_{2}\right)\left(f-a_{3}\right)\left(f-a_{4}\right)}\right) \\
& +(A+B)_{\theta-\varepsilon, \theta+\varepsilon} \\
& \times\left(r, \frac{f^{\prime}}{\left(f-a_{1}\right)\left(f-a_{2}\right)\left(f-a_{3}\right)}\right) \\
& +(A+B)_{\theta-\varepsilon, \theta+\varepsilon} \\
& \times\left(r, \frac{f^{\prime}}{\left(f-a_{1}\right)\left(f-a_{2}\right)\left(f-a_{3}\right)\left(f-a_{4}\right)}\right) \\
& +(A+B)_{\theta-\varepsilon, \theta+\varepsilon} \\
& \times\left(r, \frac{f^{\prime} P_{1}(f)}{\left(f-a_{1}\right)\left(f-a_{2}\right)\left(f-a_{3}\right)\left(f-a_{4}\right)}\right) \\
& +(A+B)_{\theta-\varepsilon, \theta+\varepsilon} \\
& \times\left(r, \frac{g^{\prime}}{\left(g-a_{2}\right)\left(g-a_{3}\right)\left(g-a_{4}\right)}\right) \\
& +(A+B)_{\theta-\varepsilon, \theta+\varepsilon} \\
& \times\left(r, \frac{g^{\prime}}{\left(g-a_{1}\right)\left(g-a_{2}\right)\left(g-a_{3}\right)}\right) \\
& +(A+B)_{\theta-\varepsilon, \theta+\varepsilon} \\
& \times\left(r, \frac{g^{\prime}}{\left(g-a_{1}\right)\left(g-a_{2}\right)\left(g-a_{3}\right)\left(g-a_{4}\right)}\right) \\
& +(A+B)_{\theta-\varepsilon, \theta+\varepsilon}
\end{aligned}
$$

$$
\begin{aligned}
& \quad \times\left(r, \frac{g^{\prime} P_{2}(g)}{\left(g-a_{1}\right)\left(g-a_{2}\right)\left(g-a_{3}\right)\left(g-a_{4}\right)}\right) \\
& +O(1) \\
& =R_{\theta-\varepsilon, \theta+\varepsilon}(r, f)+R_{\theta-\varepsilon, \theta+\varepsilon}(r, g),
\end{aligned}
$$

where $P_{1}(f)$ is a polynomial of degree no more than 2 in $f$ and $P_{2}(g)$ is a polynomial of degree no more than 2 in $g$.

Thus, we complete the proof of this lemma.

\section{Proof of Theorem 8}

Proof. Since $f$ is a meromorphic function of infinite order $\rho(r)$ and $\arg z=\theta(0 \leq \theta<2 \pi)$ is one Borel direction of $\rho(r)$ order of the meromorphic function $f$, by Lemma 16, we can get for any $\varepsilon(0<\varepsilon<\pi)$

$$
\limsup _{r \rightarrow \infty} \frac{\log S_{\theta-\varepsilon, \theta+\varepsilon}(r, f)}{\rho(r) \log r}=1
$$

And since $g \in M(\rho(r))$, we have

$$
\limsup _{r \rightarrow \infty} \frac{\log S_{\theta-\varepsilon, \theta+\varepsilon}(r, g)}{\rho(r) \log r} \leq 1
$$

Set $R(r)=O(\rho(r) \log r)$ as $r \rightarrow \infty, r \notin E$ where $E$ is a set of finite linear measure; then $R(r)=o\left(S_{\theta-\varepsilon, \theta+\varepsilon}(r, f)\right)$ as $r \rightarrow \infty$, $r \notin E$.

Since $f$ and $g$ share four distinct values $a_{j}(j=1,2,3,4)$ IM in $\Omega(\theta-\varepsilon, \theta+\varepsilon)$, suppose that $f \not \equiv g$ and none of the $a_{j}(j=1,2,3,4)$ is $\infty$. By Lemma 11, we have

$$
\begin{aligned}
2 S_{\theta-\varepsilon, \theta+\varepsilon}(r, f) \leq & \sum_{j=1}^{4} \bar{C}_{\theta-\varepsilon, \theta+\varepsilon}\left(r, \frac{1}{f-a_{j}}\right)+R_{\theta-\varepsilon, \theta+\varepsilon}(r, f) \\
\leq & C_{\theta-\varepsilon, \theta+\varepsilon}\left(r, \frac{1}{f-g}\right)+R_{\theta-\varepsilon, \theta+\varepsilon}(r, f) \\
\leq & S_{\theta-\varepsilon, \theta+\varepsilon}(r, f)+S_{\theta-\varepsilon, \theta+\varepsilon}(r, g) \\
& +R_{\theta-\varepsilon, \theta+\varepsilon}(r, f)
\end{aligned}
$$

and by interchanging $f$ and $g$, we can get that

$$
\begin{aligned}
& S_{\theta-\varepsilon, \theta+\varepsilon}(r, f)=S_{\theta-\varepsilon, \theta+\varepsilon}(r, g)+R_{\theta-\varepsilon, \theta+\varepsilon}(r, f) \\
& S_{\theta-\varepsilon, \theta+\varepsilon}(r, g)=S_{\theta-\varepsilon, \theta+\varepsilon}(r, f)+R_{\theta-\varepsilon, \theta+\varepsilon}(r, g) .
\end{aligned}
$$

Thus, it follows $R_{\theta-\varepsilon, \theta+\varepsilon}(r, g)=R_{\theta-\varepsilon, \theta+\varepsilon}(r, f)=R(r)$ and

$$
\sum_{j=1}^{4} \bar{C}_{\theta-\varepsilon, \theta+\varepsilon}\left(r, \frac{1}{f-a_{j}}\right)=2 S_{\theta-\varepsilon, \theta+\varepsilon}(r, f)+R(r) .
$$


Let $\Psi$ be the function expressed in Lemma 18. Then, $\Psi \not \equiv$ 0 . By Lemma 11 and (24), for any $b_{j} \in S(j=1,2, \ldots, m)$, we have

$$
\begin{aligned}
3 S_{\theta-\varepsilon, \theta+\varepsilon}(r, f) \leq & \sum_{j=1}^{4} \bar{C}_{\theta-\varepsilon, \theta+\varepsilon}\left(r, \frac{1}{f-a_{j}}\right) \\
& +\bar{C}_{\theta-\varepsilon, \theta+\varepsilon}\left(r, \frac{1}{f-b_{j}}\right)+R_{\theta-\varepsilon, \theta+\varepsilon}(r, f) \\
\leq & 2 S_{\theta-\varepsilon, \theta+\varepsilon}(r, f)+\bar{C}_{\theta-\varepsilon, \theta+\varepsilon}\left(r, \frac{1}{f-b_{j}}\right) \\
& +R(r) ;
\end{aligned}
$$

that is,

$$
\bar{C}_{\theta-\varepsilon, \theta+\varepsilon}\left(r, \frac{1}{f-b_{j}}\right)=S_{\theta-\varepsilon, \theta+\varepsilon}(r, f)+R(r) .
$$

Similarly, we have

$$
\bar{C}_{\theta-\varepsilon, \theta+\varepsilon}\left(r, \frac{1}{g-b_{j}}\right)=S_{\theta-\varepsilon, \theta+\varepsilon}(r, g)+R(r) .
$$

From (26) and (27), it follows

$$
\begin{aligned}
& (A+B)_{\theta-\varepsilon, \theta+\varepsilon}\left(r, \frac{1}{f-b_{j}}\right)=R(r), \\
& (A+B)_{\theta-\varepsilon, \theta+\varepsilon}\left(r, \frac{1}{g-b_{j}}\right)=R(r),
\end{aligned}
$$

for any $b_{j} \in S(j=1,2, \ldots, m)$.

Set

$$
\begin{aligned}
& \Psi_{1}:=\frac{\left(g-b_{1}\right) \cdots\left(g-b_{m}\right)}{\left(f-b_{1}\right) \cdots\left(f-b_{m}\right)} \cdot\left(\frac{g^{\prime}(f-g)}{\left(g-a_{1}\right) \cdots\left(g-a_{4}\right)}\right)^{m}, \\
& \Psi_{2}:=\frac{\left(f-b_{1}\right) \cdots\left(f-b_{m}\right)}{\left(g-b_{1}\right) \cdots\left(g-b_{m}\right)} \cdot\left(\frac{f^{\prime}(f-g)}{\left(f-a_{1}\right) \cdots\left(f-a_{4}\right)}\right)^{m} .
\end{aligned}
$$

By Lemma 17 and (28), we can get that

$$
\begin{aligned}
& (A+B)_{\theta-\varepsilon, \theta+\varepsilon}\left(r, \frac{1}{f-b_{j}} \cdot \frac{g^{\prime}(f-g)\left(g-b_{j}\right)}{\left(g-a_{1}\right) \cdots\left(g-a_{4}\right)}\right)=R(r), \\
& (A+B)_{\theta-\varepsilon, \theta+\varepsilon}\left(r, \frac{1}{g-b_{j}} \cdot \frac{f^{\prime}(f-g)\left(f-b_{j}\right)}{\left(f-a_{1}\right) \cdots\left(f-a_{4}\right)}\right)=R(r) .
\end{aligned}
$$

Thus, it follows that $(A+B)_{\theta-\varepsilon, \theta+\varepsilon}\left(r, \Psi_{j}\right)=R(r), j=1,2$. From (28), we see that "almost all" of poles and $b_{j}$-points of $f$ and $g$ in the angular domain $\Omega(\theta-\varepsilon, \theta+\varepsilon)$ are simple. Since $f, g$ share the four distinct values $a_{j}, j=1,2,3,4$ in the angular domain $\Omega(\theta-\varepsilon, \theta+\varepsilon)$ and $\bar{E}(S, \Omega(\theta-\varepsilon, \theta+\varepsilon), f) \subset$ $\bar{E}(S, \Omega(\theta-\varepsilon, \theta+\varepsilon), g)$, we can easily get that $C_{\theta-\varepsilon, \theta+\varepsilon}\left(r, \Psi_{1}\right)=$ $R(r)$. Therefore, we have

$$
S_{\theta-\varepsilon, \theta+\varepsilon}\left(r, \Psi_{1}\right)=R(r)
$$

Since $\Psi_{1} \Psi_{2} \equiv \Psi^{m}$, we can have

$$
S_{\theta-\varepsilon, \theta+\varepsilon}\left(r, \Psi_{2}\right)=R(r) .
$$

Let $\Xi_{\Omega}^{p q}\left(a_{j}\right)$ be the set of those $a_{j}$-points of $f$ and $g$ in the angular domain $\Omega(\theta-\varepsilon, \theta+\varepsilon)$ in which the multiplicities of $f$ and $g$ at these points are $p$ and $q$, respectively. For any $z_{0} \in$ $\Xi_{\Omega}^{p q}\left(a_{1}\right)$, by simple computation, we have

$$
\begin{aligned}
& \Psi_{1}\left(z_{0}\right)=\left(q \cdot \frac{f^{\prime}\left(z_{0}\right)-g^{\prime}\left(z_{0}\right)}{\left(a_{1}-a_{2}\right)\left(a_{1}-a_{3}\right)\left(a_{1}-a_{4}\right)}\right)^{m}, \\
& \Psi_{2}\left(z_{0}\right)=\left(p \cdot \frac{f^{\prime}\left(z_{0}\right)-g^{\prime}\left(z_{0}\right)}{\left(a_{1}-a_{2}\right)\left(a_{1}-a_{3}\right)\left(a_{1}-a_{4}\right)}\right)^{m} .
\end{aligned}
$$

Hence,

$$
\frac{1}{q^{m}} \Psi_{1}\left(z_{0}\right)-\frac{1}{p^{m}} \Psi_{2}\left(z_{0}\right)=0
$$

Similarly, we can see that (34) holds for any $z_{0} \in \Xi_{\Omega}^{p q}\left(a_{j}\right), j=$ $2,3,4$.

Now, two cases will be considered below.

Case 1. Suppose that $\Psi^{p q}:=\left(1 / q^{m}\right) \Psi_{1}-\left(1 / p^{m}\right) \Psi_{2} \not \equiv 0$, for all positive integers $p, q$.

First, we use $C_{\theta-\varepsilon, \theta+\varepsilon}^{p q}\left(r, 1 /\left(f-a_{j}\right)\right)$ to denote the counting function of $f$ in $\Omega(\theta-\varepsilon, \theta+\varepsilon)$ with respect to the set $\Xi_{\Omega}^{p q}\left(a_{j}\right)$, and we also use $\bar{C}_{\theta-\varepsilon, \theta+\varepsilon}^{p q}\left(r, 1 /\left(f-a_{j}\right)\right)$ to denote the corresponding reduced counting function. Thus, we have

$$
\begin{aligned}
& C_{\theta-\varepsilon, \theta+\varepsilon}\left(r, \frac{1}{f-a_{j}}\right)=\sum_{p, q=1}^{\infty} C_{\theta-\varepsilon, \theta+\varepsilon}^{p q}\left(r, \frac{1}{f-a_{j}}\right), \\
& \bar{C}_{\theta-\varepsilon, \theta+\varepsilon}\left(r, \frac{1}{f-a_{j}}\right)=\sum_{p, q=1}^{\infty} \bar{C}_{\theta-\varepsilon, \theta+\varepsilon}^{p q}\left(r, \frac{1}{f-a_{j}}\right) .
\end{aligned}
$$

From the above two equations and (28)-(32), we can see that $S_{\theta-\varepsilon, \theta+\varepsilon}\left(r, \Psi^{p q}\right)=R(r)$. And by (34) each zero of $f-a_{j}$ is a zero of $\Psi^{p q}$, so with the help of Lemma 10 and $\Psi^{p q} \not \equiv 0$, we can get

$$
\begin{aligned}
\bar{C}_{\theta-\varepsilon, \theta+\varepsilon}^{p q}\left(r, \frac{1}{f-a_{j}}\right) & \leq \bar{C}_{\theta-\varepsilon, \theta+\varepsilon}^{p q}\left(r, \frac{1}{\Psi^{p q}}\right) \leq S_{\theta-\varepsilon, \theta+\varepsilon}\left(r, \frac{1}{\Psi^{p q}}\right) \\
& \leq S_{\theta-\varepsilon, \theta+\varepsilon}\left(r, \Psi^{p q}\right)+O(1)=R(r),
\end{aligned}
$$


for some $p, q$. Since $S_{\theta-\varepsilon, \theta+\varepsilon}(r, f)=S_{\theta-\varepsilon, \theta+\varepsilon}(r, g)+R(r)$, it follows

$$
\begin{aligned}
& \bar{C}_{\theta-\varepsilon, \theta+\varepsilon}\left(r, \frac{1}{f-a_{j}}\right)= \sum_{\max (p, q) \geq 5} \bar{C}_{\theta-\varepsilon, \theta+\varepsilon}^{p q}\left(r, \frac{1}{f-a_{j}}\right) \\
&+R(r, f) \\
& \leq \frac{1}{5} \sum_{\max (p, q) \geq 5} C_{\theta-\varepsilon, \theta+\varepsilon}^{p q}\left(r, \frac{1}{f-a_{j}}\right) \\
&+\frac{1}{5} \sum_{\max (p, q) \geq 5} C_{\theta-\varepsilon, \theta+\varepsilon}^{p q}\left(r, \frac{1}{g-a_{j}}\right) \\
&+R(r) \quad j=1,2,3,4 . \\
& \leq \frac{1}{5}\left(C_{\theta-\varepsilon, \theta+\varepsilon}\left(r, \frac{1}{f-a_{j}}\right)\right. \\
& \leq \frac{2}{5} S_{\theta-\varepsilon, \theta+\varepsilon}(r, f)+R(r), \\
&\left.+C_{\theta-\varepsilon, \theta+\varepsilon}\left(r, \frac{1}{g-a_{j}}\right)\right)+R(r) \\
& \quad j
\end{aligned}
$$

From the above inequality and (24), we can get

$$
2 S_{\theta-\varepsilon, \theta+\varepsilon}(r, f) \leq \frac{8}{5} S_{\theta-\varepsilon, \theta+\varepsilon}(r, f)+R(r) .
$$

Since $f$ is of infinite order $\rho(r)$ and $R(r)=O(\rho(r) \log r)$, we can get a contradiction to (38).

Case 2. Suppose that $\Psi^{p q}:=\left(1 / q^{m}\right) \Psi_{1}-\left(1 / p^{m}\right) \Psi_{2} \equiv 0$, for some positive integers $p, q$. From the definitions of $\Psi_{1}$ and $\Psi_{2}$, we have

$$
\begin{aligned}
\left(\frac{p}{q}\right)^{m} & \cdot \frac{\left(g-b_{1}\right)^{2} \cdots\left(g-b_{m}\right)^{2}}{\left(f-b_{1}\right)^{2} \cdots\left(f-b_{m}\right)^{2}} \\
& \equiv\left(\frac{f^{\prime}\left(g-a_{1}\right) \cdots\left(g-a_{4}\right)}{g^{\prime}\left(f-a_{1}\right) \cdots\left(f-a_{4}\right)}\right)^{m} .
\end{aligned}
$$
tion.

Next, we take the following two subcases into considera-

Subcase 2.1. Suppose that $p \neq q$. Without loss of generality, we may assume that $p<q$. For some two positive integers $p_{1}$ and $q_{1}$, if $z_{1} \in \Xi_{\Omega}^{p_{1} q_{1}}\left(a_{j}\right)$ for some $j \in\{1,2,3,4\}$, then (39) implies that $p / q=p_{1} / q_{1}$. Hence, $q_{1}>p_{1} \geq 1$, and $q_{1} \geq 2$ which means that any $a_{j}$-points $(j=1,2,3,4)$ of $g$ in $\Omega(\theta-\varepsilon, \theta+\varepsilon)$ are multiple. By Lemma 11 and (24), we can get

$$
\begin{aligned}
2 S_{\theta-\varepsilon, \theta+\varepsilon}(r, g) & =\sum_{j=1}^{4} \bar{C}_{\theta-\varepsilon, \theta+\varepsilon}\left(r, \frac{1}{g-a_{j}}\right)+R(r) \\
& \leq \frac{1}{2} \sum_{j=1}^{4} C_{\theta-\varepsilon, \theta+\varepsilon}\left(r, \frac{1}{g-a_{j}}\right)+R(r) \\
& \leq 2 S_{\theta-\varepsilon, \theta+\varepsilon}(r, g)+R(r) ;
\end{aligned}
$$

it follows that

$$
\begin{gathered}
S_{\theta-\varepsilon, \theta+\varepsilon}(r, g)=C_{\theta-\varepsilon, \theta+\varepsilon}\left(r, \frac{1}{g-a_{j}}\right)+R(r), \\
C_{\theta-\varepsilon, \theta+\varepsilon}\left(r, \frac{1}{g-a_{j}}\right)=2 \bar{C}_{\theta-\varepsilon, \theta+\varepsilon}\left(r, \frac{1}{g-a_{j}}\right)+R(r) .
\end{gathered}
$$

From (41) and (42), we can see that "almost all" of $a_{j}$ points of $g$ have multiplicity 2, and "almost all" of $a_{j}$-points of $f$ are simple in $\Omega(\theta-\varepsilon, \theta+\varepsilon)$. Without loss of generality, we may assume that $f$ and $g$ attain the values $a_{3}$ and $a_{4}$ in $\Omega(\theta-\varepsilon, \theta+\varepsilon)$. Set

$$
\begin{aligned}
\Phi_{1}:= & \frac{2 f^{\prime}\left(f-a_{4}\right)}{\left(f-a_{1}\right)\left(f-a_{2}\right)\left(f-a_{3}\right)} \\
& -\frac{g^{\prime}\left(g-a_{4}\right)}{\left(g-a_{1}\right)\left(g-a_{2}\right)\left(g-a_{3}\right)}, \\
\Phi_{2}:= & \frac{2 f^{\prime}\left(f-a_{3}\right)}{\left(f-a_{1}\right)\left(f-a_{2}\right)\left(f-a_{4}\right)} \\
& -\frac{g^{\prime}\left(g-a_{3}\right)}{\left(g-a_{1}\right)\left(g-a_{2}\right)\left(g-a_{4}\right)} .
\end{aligned}
$$

Since $\Phi_{i}(i=1,2)$ is analytic at the poles of $f$ and of $g$ and also at those common $a_{j}$-points of $f$ and $g$ which have multiplicity 1 with respect to $f$ and multiplicity 2 with respect to $g$, by Lemma 17, we have $S_{\theta-\varepsilon, \theta+\varepsilon}\left(r, \Phi_{i}\right)=R(r), i=1,2$. If $\Phi_{i} \not \equiv 0$, then $C_{\theta-\varepsilon, \theta+\varepsilon}\left(r, 1 /\left(g-a_{4}\right)\right) \leq C_{\theta-\varepsilon, \theta+\varepsilon}\left(r, 1 / \phi_{1}\right)=R(r)$, which contradicts to (41). Then, $\Phi_{1} \equiv 0$. Similarly, we have $\Phi_{2} \equiv 0$. Therefore, from the definitions of $\Phi_{1}$ and $\Phi_{2}$, we have

$$
\left(\frac{f-a_{4}}{f-a_{3}}\right)^{2} \equiv\left(\frac{g-a_{4}}{g-a_{3}}\right)^{2}
$$

Since $f \not \equiv g$, from (44), we have

$$
\frac{f-a_{4}}{f-a_{3}} \equiv-\frac{g-a_{4}}{g-a_{3}},
$$

which implies that $f$ and $g$ share $a_{3}, a_{4} \mathrm{CM}$ in $\Omega(\theta-\varepsilon, \theta+\varepsilon)$. Since $f$ and $g$ assume the value $a_{3}$, there exist positive integers 
$p_{1}, q_{1}$ such that $\Xi_{\Omega}^{p_{1} q_{1}}\left(a_{3}\right) \neq \emptyset$. From the considerations above we get $q_{1}>p_{1}$, contradicting the fact that $f$ and $g$ share $a_{3}$ CM.

Subcase 2.2. Suppose that $p=q$.

In this subcase, (39) becomes

$$
\frac{\left(g-b_{1}\right)^{2} \cdots\left(g-b_{m}\right)^{2}}{\left(f-b_{1}\right)^{2} \cdots\left(f-b_{m}\right)^{2}} \equiv\left(\frac{f^{\prime}\left(g-a_{1}\right) \cdots\left(g-a_{4}\right)}{g^{\prime}\left(f-a_{1}\right) \cdots\left(f-a_{4}\right)}\right)^{m} \text {. }
$$

which implies that $f$ and $g$ share the four values $a_{j}(j=$ $1,2,3,4) \mathrm{CM}$ in $\Omega(\theta-\varepsilon, \theta+\varepsilon)$. Then by applying Theorem 7 , $g$ is a Möbius transformation of $f$. Furthermore, two of the four values, say $a_{1}, a_{2}$, are Picard exceptional values of $f$ and $g$ in $\Omega(\theta-\varepsilon, \theta+\varepsilon)$. Set

$$
\begin{aligned}
\Lambda_{1}:= & \frac{f^{\prime}\left(f-a_{4}\right)}{\left(f-a_{1}\right)\left(f-a_{2}\right)\left(f-a_{3}\right)} \\
& -\frac{g^{\prime}\left(g-a_{4}\right)}{\left(g-a_{1}\right)\left(g-a_{2}\right)\left(g-a_{3}\right)}, \\
\Lambda_{2}:= & \frac{f^{\prime}\left(f-a_{3}\right)}{\left(f-a_{1}\right)\left(f-a_{2}\right)\left(f-a_{4}\right)} \\
& -\frac{g^{\prime}\left(g-a_{3}\right)}{\left(g-a_{1}\right)\left(g-a_{2}\right)\left(g-a_{4}\right)} .
\end{aligned}
$$

Similar to the discussion in Subcase 2.1 for $\Lambda_{1}, \Lambda_{2}$, we can get

$$
\frac{f-a_{3}}{f-a_{4}} \equiv-\frac{g-a_{3}}{g-a_{4}} .
$$

We define the Möbius transformations $T, H$, and $J$ by

$$
T(\omega):=\frac{w-a_{3}}{w-a_{4}}, \quad H(w):=-w, \quad J:=T^{-1} \circ H \circ T .
$$

Then, we have

$$
T \circ f=-T \circ g ; \quad \text { hence, } g=J \circ f \text {. }
$$

Obviously, $a_{3}$ and $a_{4}$ are the fixed points of $J$. Therefore, there exist no fixed points of $J$ in the set $S$. Let some $b \in S$ be given. Then, in vies of $b \neq a_{1}, a_{2}$, there exists a $z_{0} \in \mathbb{C}$ such that $b=$ $f\left(z_{0}\right)$, and from $\bar{E}(S, \Omega(\theta-\varepsilon, \theta+\varepsilon), f) \subseteq \bar{E}(S, \Omega(\theta-\varepsilon, \theta+\varepsilon), g)$ we obtain

$$
J(b)=J\left(f\left(z_{0}\right)\right)=g\left(z_{0}\right) \in S .
$$

So $S$ is invariant under $J$. Furthermore, we have $J \circ J=I$ where $I$ denotes the identical transformation. Hence, $S$ must contain an even number of values. Thus, the proof of Theorem 8 is completed.

\section{Acknowledgments}

This project is supported by the National Natural Science Foundation of China (project nos. 11171184, 61202313) and the Natural Science Foundation of Jiangxi Province in China (nos. 2010GQS0119 and 20132BAB211001).

\section{References}

[1] W. K. Hayman, Meromorphic Functions, Oxford University Press, London, UK, 1964.

[2] L. Yang, Value Distribution Theory, Springer/Science Press, Berlin, Germany, 1993.

[3] W. C. Lin, S. Mori, and K. Tohge, "Uniqueness theorems in an angular domain," The Tohoku Mathematical Journal, vol. 58, no. 4, pp. 509-527, 2006.

[4] R. Nevanlinna, Le Théorème de Picard-Borel et la Théorie des Fonctions Méromorphes, Chelsea Publishing, New York, NY, USA, 1974, (Frensh).

[5] H.-X. Yi and C.-C. Yang, Uniqueness Theory of Meromorphic Functions, vol. 557, Kluwer Academic, Dordrecht, The Netherlands, 2003, Chinese Original: Science Press, Beijing, China, 1995.

[6] J.-H. Zheng, "On uniqueness of meromorphic functions with shared values in some angular domains," Canadian Mathematical Bulletin. Bulletin Canadien de Mathématiques, vol. 47, no. 1, pp. 152-160, 2004.

[7] J.-H. Zheng, "On uniqueness of meromorphic functions with shared values in one angular domain," Complex Variables. Theory and Application, vol. 48, no. 9, pp. 777-785, 2003.

[8] T.-B. Cao and H.-X. Yi, "On the uniqueness of meromorphic functions that share four values in one angular domain," Journal of Mathematical Analysis and Applications, vol. 358, no. 1, pp. 8197, 2009.

[9] Z.-J. Wu, "On uniqueness of meromorphic functions in an angular domain," Kodai Mathematical Journal, vol. 30, no. 3, pp. 352-360, 2007.

[10] Z.-J. Wu and D.-C. Sun, "A remark on uniqueness theorems in an angular domain," Japan Academy. Proceedings A, vol. 84, no. 6, pp. 73-77, 2008.

[11] H.-Y. Xu and T.-B. Cao, "Uniqueness of two analytic functions sharing four values in an angular domain," Annales Polonici Mathematici, vol. 99, no. 1, pp. 55-65, 2010.

[12] H.-Y. Xu and T.-B. Cao, "Analytic functions in the unit disc sharing values in a sector," Annales Polonici Mathematici, vol. 103, no. 3, pp. 263-275, 2012.

[13] M. L. Cartwright, "On the directions of borel of functions which are regular and of finite order in an angle," Proceedings of the London Mathematical Society, vol. S2-38, no. 1, pp. 503-541.

[14] W. K. Hayman and S. J. Wu, "Value distribution theory and the research of Yang Lo," Science in China B, vol. 53, no. 3, pp. 513522,2010

[15] C. N. Linden, "On a conjecture of Valiron concerning sets of indirect Borel points," Journal of the London Mathematical Society, vol. 41, pp. 304-312, 1966.

[16] G. Valiron, "Entire functions and Borel's directions," Proceedings of the National Academy of Sciences of the United States of America, vol. 20, no. 3, pp. 211-215, 1934.

[17] S. J. Wu, "Further results on Borel removable sets of entire functions," Annales Academiae Scientiarum Fennicae A, vol. 19, no. 1, pp. 67-81, 1994.

[18] S. J. Wu, "The distribution of the Borel directions of entire functions," Chinese Annals of Mathematics A, vol. 14, no. 4, pp. 400-406, 1993.

[19] L. Yang, "Borel directions of meromorphic functions in an angular domain," Scientia Sinica. Zhongguo Kexue, pp. 149-164, 1979. 
[20] L. Yang and G. H. Zhang, "Distribution of Borel directions of entire functions," Acta Mathematica Sinica. Shuxue Xuebao, vol. 19, no. 3, pp. 157-168, 1976.

[21] L. Yang and G. H. Zhang, "Progress in the value distribution theory of meromorphic functions," Kexue Tongbao. Chinese Science Bulletin, vol. 22, no. 9, pp. 375-380, 1977.

[22] L. Yang and K. H. Chang, "Sur la distribution des directions de Borel des fonctions méromorphes," Scientia Sinica, vol. 16, pp. 465-482, 1973.

[23] G. H. Zhang, "Common Borel directions of meromorphic functions and their successive derivatives or integrals. III," Acta Mathematica Sinica. Shuxue Xuebao, vol. 20, no. 4, pp. 237-247, 1977.

[24] Q. D. Zhang, "T direction and Borel direction of meromorphic functions of finite and positive order," Acta Mathematica Sinica. Chinese Series, vol. 50, no. 2, pp. 413-419, 2007.

[25] C. T. Chuang, Singular Direction of Meromorpic Functions, Science Press, Beijing, China, 1982.

[26] J. R. Long and P. C. Wu, "Borel directions and uniqueness of meromorphic functions," Chinese Annals of Mathematics A, vol. 33, no. 3, pp. 261-266, 2012.

[27] A. A. Goldberg and I. V. Ostrovskiur, The Distribution of Values of Meromorphic Function, Nauka, Moscow, 1970, (Russian).

[28] A. A. Goldberg, "Nevanlinna's lemma on the logarithmic derivative of a meromorphic function," Mathematical Notes, vol. 17, no. 4, pp. 310-312, 1975.

[29] C. T. Chuang, "On Borel directions of meromorphic functions of infinite order. II," Bulletin of the Hong Kong Mathematical Society, vol. 2, no. 2, pp. 305-323, 1999. 


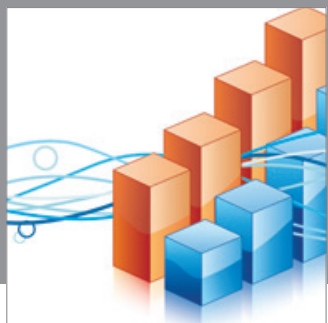

Advances in

Operations Research

mansans

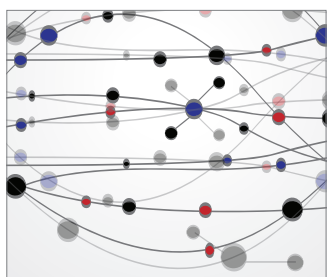

The Scientific World Journal
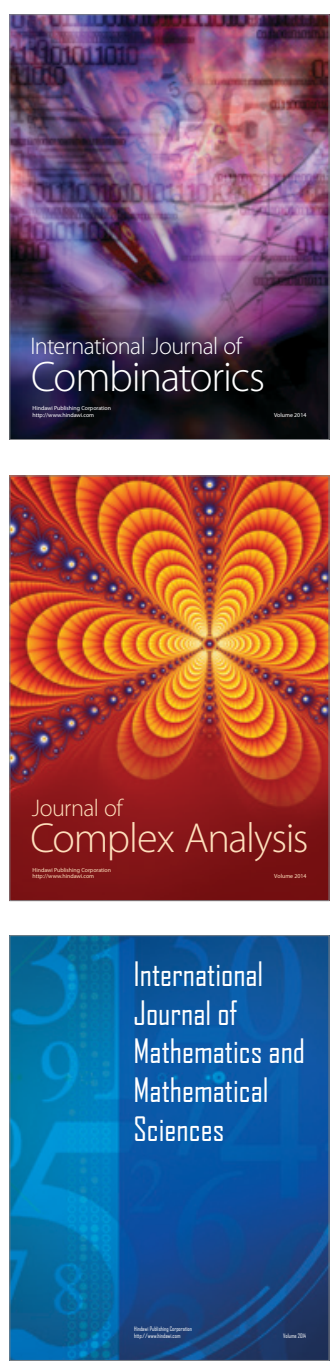
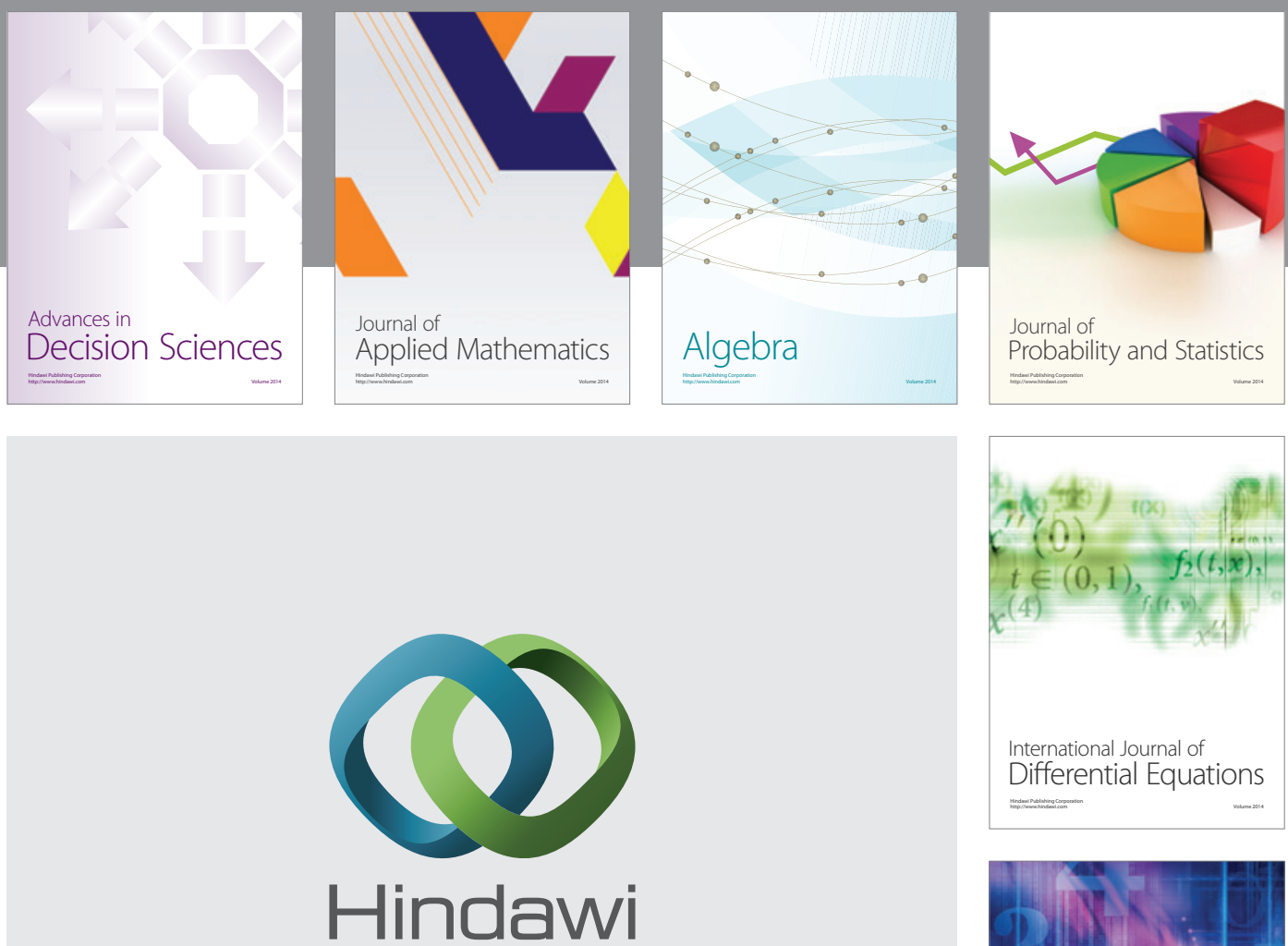

Submit your manuscripts at http://www.hindawi.com
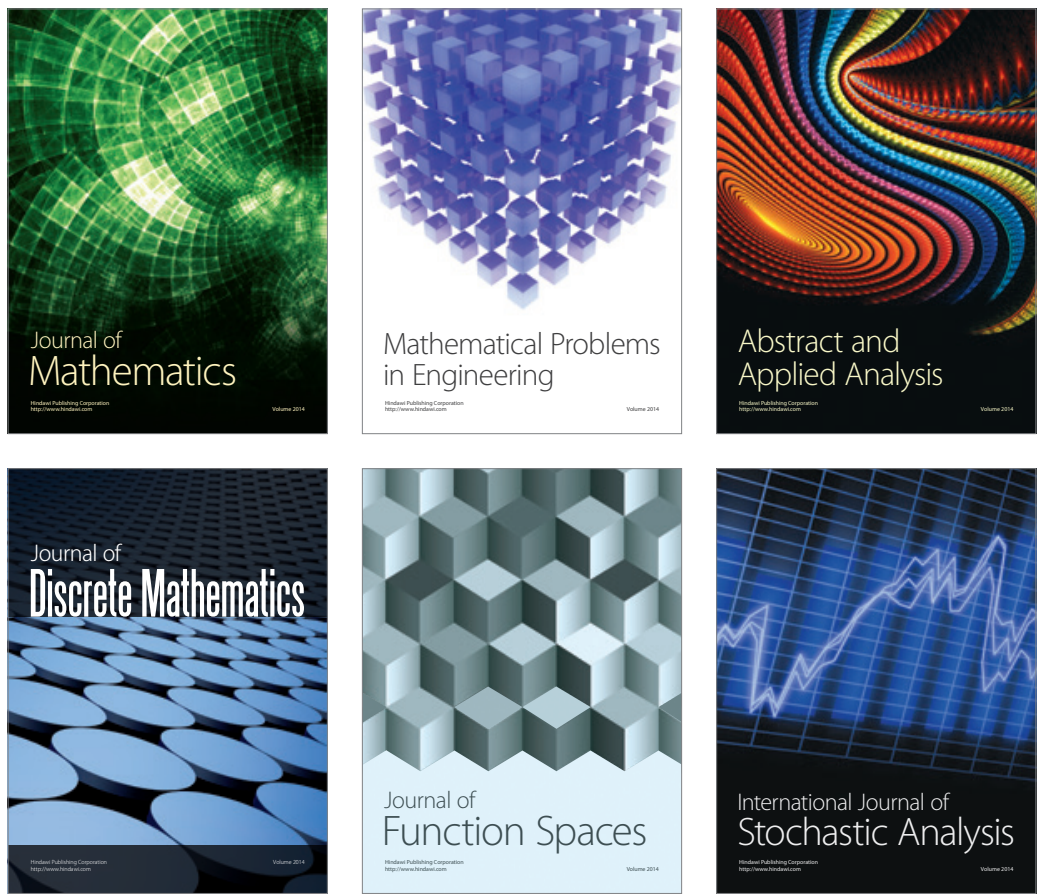

Journal of

Function Spaces

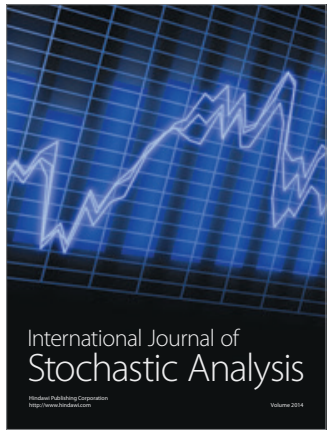

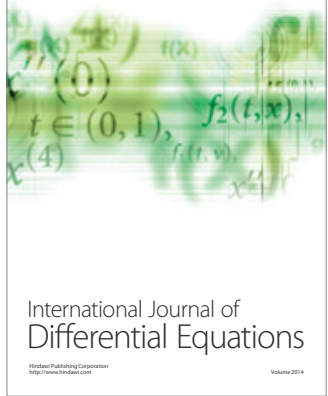
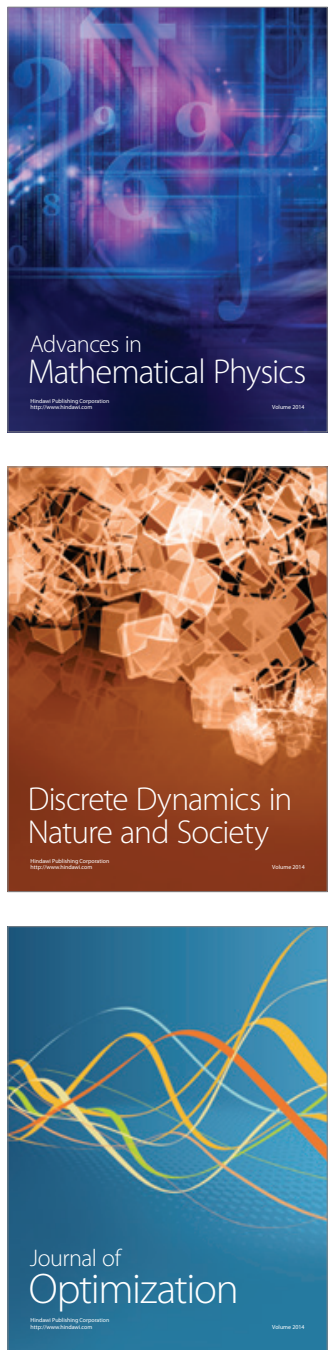